\title{
Tidal Force of Sun Due to Planetary Radial ALIGNMENT AND SUN-SPOT CYCLE
}

\author{
S.D. Verma \\ Department of Physics and Space Sciences, Gujarat University, \\ Ahmedabad 380009, India
}

\begin{abstract}
It is well known that the Sun's radiation and a large number of phenomena occurring on the sun have influence on the Earth's near environment i.e. A tmosphere, Iono sphere,Magnetosphere, etc. These manifest themselves as day-night, seasons, tides and many changes in the neutral atmosphere; changes in meteorological parameters. These changes are directly or indirectly related to variations in solar pardmeters, such as solar flares, magnetic storms, variations in sunspot number occurring in solar photosphere. Sunsports are observed, their number counted and their accurate records maintained for long time (many centuaries). The sunspot number seems to follow periodic changes with several periods; mainly 11 years and 23.5 years. Recently it has been shown* that the combined $t i-$ dal force of the inner planets and two largest iplanets, Jupiter and Saturn, have periodic change of 11 and 23.5 years. It was proposed that this small force may be having a tiny influence on the surface of the sun and causing some nonlinear effect which results into formation of sunspots and thus causes the variations in the number of sunspots. In the present work it is shown that whenever the combined tidal force on sun increases then sunspot number seems to increase and when force decreases sunspot number decreases. This is shown for Solar Cycle number 21 .
\end{abstract}

* "Influence of planetary motion and Radial alignment of planets on Sun" S.D. Verma publ ished in "Space Dynamics and Celestial Mechanics", Ed.K.B. Bhatnagar,p.143-154,1986 by D.Reidel Publishing Company. 


\section{INTRODUCT ION}

Sunspots have been observed for hundreds of years and records are made. The longest and most reliable records begin from 1850 A.D. onwards, eventhough the early telescopic observations started in $1610 \mathrm{~A} . \mathrm{D}$. The sunspot cycle of 11 years period has been known since very early times. The sunspots appear on the photospheric disc only in the equatorial region, within 400 North and South latitudes. In order to put the real-time monitoring of the sunspot activity on a quantitative basis, an arbitrary index $\bar{R}$ was established which is still used. A daily measure of the spottiness is represented by a Zurich Sunspot number ' $\bar{R}$ ' defined as

$$
\bar{R}=k(10 g+f)
$$

where $f$ is the number of individual spots, $g$ the number of recognizable group of spots, and $k$ a subjective correction factor to allow for differences in observing conditions of tel escopes or observers. The value of $\bar{R}$ has stood up reasonably well in comparison with related more objective indices such as the $10.7 \mathrm{MHz}$ solar radio flux on daily measurements of sunspot areas. In Fig. $1 \bar{R}$ is plotted versus time for the last 200 years, taken from Eddy (1980), which clearly indicates cyclic variations of approximately 11 year period. Currie (1973) has applied the new data adaptive approach to power spectrum estimation of studying various 1 ines (periodicities) present in the sunspot number variations. These lines are discussed and explained in the paper.

A plot of the monthly average sunspot number $\bar{R}$ versus time, shown in Fig. 2, for the present solar cycle (cycle number 21 ) clearly indicates that $\bar{R}$ changes $i . e$. increases several times quite drastically within a month. Many times it decreases also quite suddenly though slightly slower than its increase. A model is proposed in the next section to explain these changes in $\bar{R}$ with time. This model islused to explain various lines (periodicities) in the power spectrum of sunspot number .

\section{MODEL}

Before giving the basic features of the model, the variation ( $i . e$. sudden changes) in $\bar{R}$ is shown in Fig. 2 . Here monthly average $\bar{R}$ is plotted vs time for several years for the present solar cycle (cycle no.21) taken from NOAA prompt report. It is worth pointing out that substantial changes in $\bar{R}$ are recorded in consecutive months. This means that sudden changes, both increase and decrease in monthly average sunspot number $\bar{R}$ occur within a month. Attempt is made to develop a basic model to explain these features. 

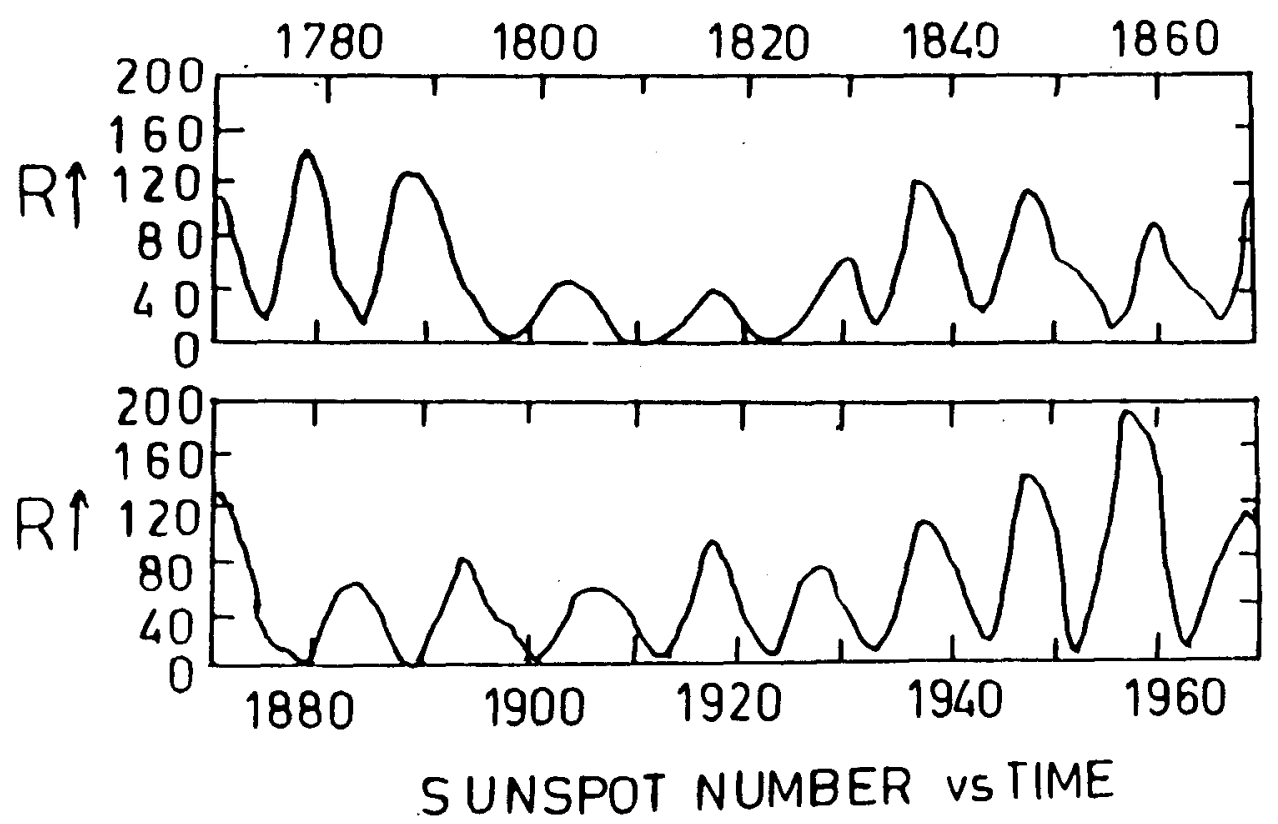

Fig. 1: Annual Mean Sunspot Number of last two hundred years.

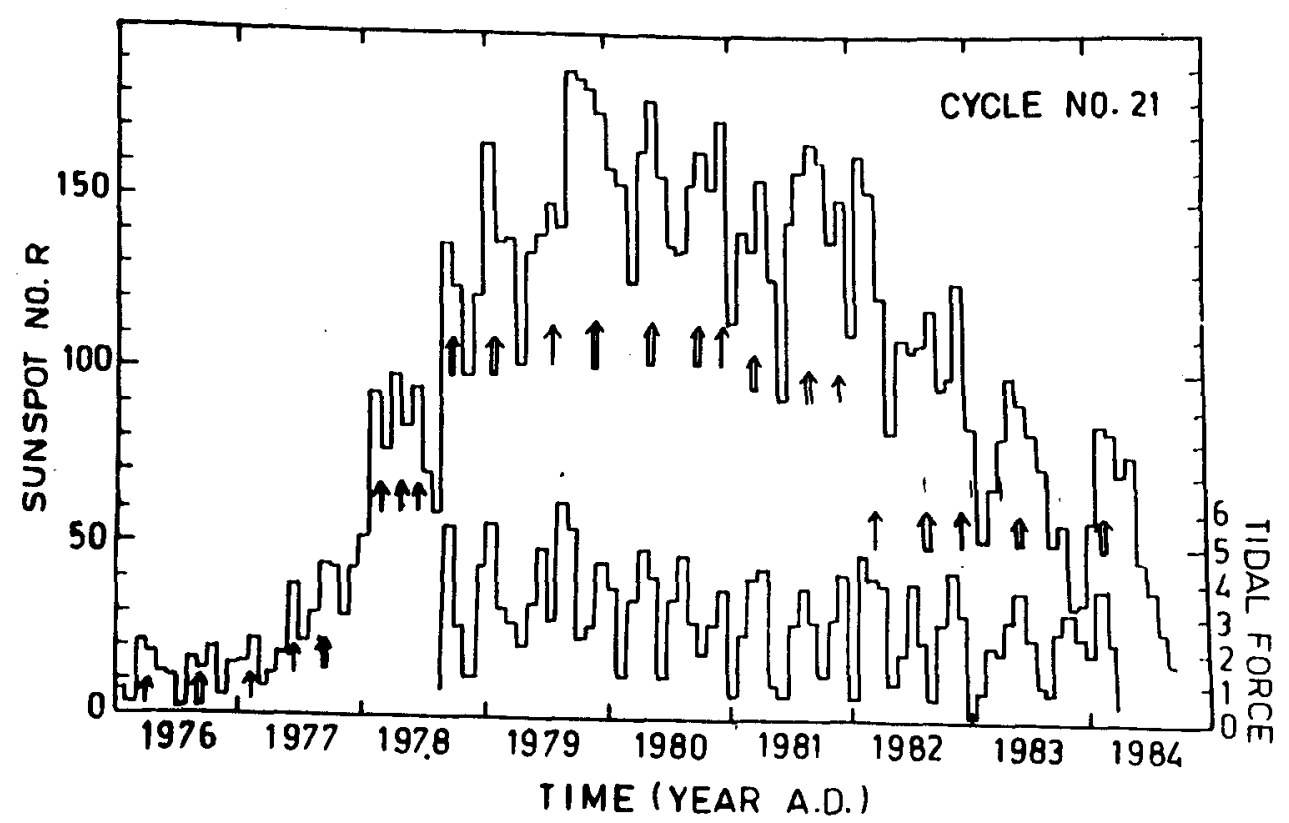

Fig. 2: Monthly arerage Sunspot number $R$ versus time for solar cycle number 21. (1976-1984 AD) 
The proposed mechanism in this model is that the differential tidal force (T.F.) of planets on Sun's surface,initiates some process which causes instabilities in the convective zone of the sun. This leads to the formation of sunspots. This tidal force per unit mass, at and near the surface of the sun due to earth is expressed below

$$
\lambda \cdot T \cdot F \cdot=4 G M_{0} R_{0} R_{0}^{-3}
$$

where $R_{0}$ is the radius of sun, G gravitational constant, $M_{0}$ the mass and $R_{0}$ the orbital radius of the planet Farth in this case.

The Table 1 below gives the value of the Differential Tidal Force (T.F.) per unit mass on the equatorial region of the surface of the sun. It is expressed as

$$
F=\left(M / M_{0}\right)\left(R / R_{0}\right)^{-3}
$$

which is normalised to the force due to Earth. Periods of the planets as well as the radial alignment period of planets with Jupiter is also given in the table.

\begin{tabular}{|c|c|c|c|c|c|c|}
\hline & Mercury & Venus & Earth & Mars & Jup iter & Saturn \\
\hline D.T.F & 0.91 & 2.16 & 1 & .03 & 2.26 & 0.11 \\
\hline $\begin{array}{l}\text { Period } \\
\text { (Years) }\end{array}$ & 0.24 & 0.615 & 1 & 1.88 & 11.86 & 29.5 \\
\hline $\begin{array}{l}\text { Align- } \\
\text { ment } \\
\text { Period }\end{array}$ & $\mathrm{T}_{\mathrm{jm}}$ & $T_{j v}$ & $\mathrm{~T}_{j e}$ & $\mathrm{~T}_{\text {jma }}$ & $\begin{array}{ll}- \\
11\end{array}$ & $T_{j s}$ \\
\hline (Years) & .246 & .645 & 1.09 & 2.29 & - & 19.83 \\
\hline
\end{tabular}

TABLE 1

This force is the largest due to Jupiter. Now whenever there is radial alignment $\left(0^{\circ}\right.$ or $\left.180^{\circ}\right)$ of one or more planets with Jupiter, then the forces due to aligned planets add up and magnitude of the force ' $F$ ' increases. The sum of $T . F$. due to Jupiter, Venus, Earth, Mercury, Saturn and Mars is evaluated and is shown in lower part of Fig. 2. This plot clearly indicates that whenever there is a radial alignment of two or more planets with Jupiter there is an increase in the magnitude of this force, shown by lower histogram in Fig. 2. Notice 
that every time there is an increase in this force, simultaneously there is an increase in $\bar{R}$. It is worth pointing-out that the peaks in $F$ precede the increase in value of $\bar{R}$ by about one month or less. Most of the sudden decreases i.e. depressions in $\overline{\mathrm{R}}$ value are also preceded by a decrease in the magnitude of the force. There is one to one correlation between magnitude of T.F. and the number $\bar{R}$. It is inferred that every time force increases sunspots are formed and $\bar{R}$ increases. The time of formation $t_{f}$ of sunspots is about a month after alignment

$$
\mathbf{t}_{\mathrm{f}} \stackrel{\sim}{<} 1 \text { month }
$$

Similarly, whenever the magnitude of T.F. decreases, it is followed by a decrease in the number $\bar{R}$. This indicates that reduction in force allows sunspots to decay (or disappear). The decrease in $\bar{R}$ is sometimes delayed by a month or more. From this it is inferred that decay of sunspot occurs in a time interval of more than a month. Let it be called as decay time, (time of disappearance) $t_{d}$. Looking at the histograms of Fig. 2 for 1978 and 1981 one notices that $t_{d}$ is more than a month,

$$
t_{d} \stackrel{\sim}{>} 1 \text { month }
$$

Thus, looking at the one to one correspondence seen in $T . F$. and $\bar{R}$, it is inferred that sunspot number is related to the tidal force, their formation is perhaps initiated by T.F. and formation time is about a month or less. While the reduction in magnitude of the force allows some sunspots to decay and disappear in time of more than a month.

This model was first proposed by Verma (1986) where attempt was made to explain the main sunspot cycle periodicities. This feature is discussed in detail in the next section.

\section{PER IODIC LINES OF SPECTRUM OF SUNSPOT NUMBER}

Using hundred year's data of sunspot number Currie (1973) obtained a Iine spectrum and found 11.1 and 23.6 years as the main periods (lines). This spectrum is reproduced and shown in Fig. 1. These periodic cycles (1ines) are naturally explained using the model of Verma (1986). The main idea of the model is represented here in the context with the periodic (cycle) lines. The tidal force of Jupiter on the sun is the largest. Fach time a planet is radially aligned with Jupiter the tidal force of the two and force increases in cycle fashion with the period of alignment of a planet with Jupiter. The alignment periods of Mercury $\left(\mathrm{T}_{\mathrm{jm}}\right)$, Venus $\left(T_{j v}\right)$, Earth $\left(T_{j e}\right)$, Mars $\left(T_{j m a}\right)$ and $\operatorname{Saturn}\left(T_{j s}\right)$ with Jupiter are evaluated and given in Table II. Let us consider the alignment period of two planets Venus and Earth with 
Jupiter. The alignment period $T_{j v e}$ will be integral multiple of $T_{j v}$ and $T_{j e}$. Similarly $T_{j v m}$, alignment period of venus and Mercury with Jupiter will be multiple of $T_{j v}$ and $T_{j m}$. When this idea is extended to radial alignment of three planets with Jupiter, then $T_{j v e m}$ will be integral multiple of $\mathrm{T}_{j v}, \mathrm{~T}_{j e}$ and $\mathrm{T}_{j m}$. This way periods are derived for one, $t$ wo, three or four planet alignments with Jupiter. These are listed in Table II. Large number of these periods are matching quite well with lines (periods) of the power spectrum of sunspots (Currie 1973), both are listed in Table II. This comparison seems to indicate good agreement between calculated and observed 1 ines (periods).

When Currie (1973) used sunspot data of about two hundred years (1770-1960) a marginal evidence is seen for a line with periodicity of 66.7 years. This period is explained quite well with the model proposed by Verma (1986), by considering radial alignment of Mercury, Venus, Earth and Mars with Jupiter and is also listed in the Table II.

The agreement between calculated and observed values is reasonable within the uncertainties of the measurements.

\section{DISCUSSION AND SUMMARY}

The basic model is based on the idea that the differential tidal force due to various planets on the surface of the sun changes with time due to the radial alignments of one or more planets with Jupiter. It is believed that this force initiates the process which creates the sunspots. Thus the time variation of this force is correlated with the changes in the sunspot number. In this paper one to one correlation is shown between the two. The comparison of the peaks and a noticeable small delay between the peaks of sunspot number and those of the force, is inferred as the time bf initiation or $t$ ime of formation of the sunspots $\left(t_{f}<a\right.$ month) and $t$ he relaxation time, after the force is suddenly reduced, is the time in which sunspots decay or disappear. This decay time, $t_{d}$ is > a month.

Various periods i.e.lines in the spectrum of sunspot number from few years to 66 years have been explained in terms of the alignment periods of one or more planets with Jupiter. Several periods of less than 2 years are predicted.

Thus there are clear indications which demonstrate that the planetary tidal force (T.F.) has influence on the Sun's surface, which is visible in the form of sunspots on the photospheric disc of sun. 
TABLE II

\begin{tabular}{|c|c|c|c|c|}
\hline $\begin{array}{l}\text { Name of } \\
\text { the } \\
\text { planets } \\
\text { aligned }\end{array}$ & $\begin{array}{l}\text { Al ignment } \\
\text { Per iod } \\
\text { (Year) }\end{array}$ & $\begin{array}{l}\text { Observed } \\
\text { l ines } \\
\text { (periods) } \\
\text { of sunspot } \\
\text { number } \\
\text { spectrum } \\
\text { in years } \\
\text { Currie(1973) }\end{array}$ & $\begin{array}{l}\text { Sum of } \\
\text { T.F. }\end{array}$ & $\begin{array}{l}\text { Period } \\
\text { evaluation }\end{array}$ \\
\hline$J+M$ & .246 & * & 3.17 & $\mathrm{~T}_{\mathrm{jm}}$ \\
\hline$J+V$ & .645 & * & 4.42 & $T_{j v}$ \\
\hline$J+E$ & 1.09 & * & 3.26 & $\mathrm{~T}_{\mathrm{je}}$ \\
\hline$J+M a$ & 2.29 & 2.27 & 2.29 & $\mathrm{~T}_{\text {jma }}$ \\
\hline $\mathrm{J}+\mathrm{S}$ & 19.83 & * & & $T_{j s}$ \\
\hline$J+V+E$ & 10.9 & 11.1 & 5.42 & $10 \mathrm{~T}_{j \mathrm{e}}=17 \mathrm{~T}_{\mathrm{jv}}$ \\
\hline \multirow[t]{5}{*}{ Harmonics } & 5.5 & 5.68 & & \\
\hline & 3.66 & 3.68 & & \\
\hline & 2.75 & 2.68 & & \\
\hline & 2.2 & 2.17 & & \\
\hline & 9.75 & 9.9 & & $9 \mathrm{~T}_{j \mathrm{e}}=15 \mathrm{~T}_{\mathrm{jv}}$ \\
\hline \multirow[t]{3}{*}{$J+V+M$} & 23.61 & 23.6 & 5.33 & $37 T_{j v}=95 T_{j m}$ \\
\hline & 14.67 & 14.7 & & $23 T_{j v}=59 T_{j m}$ \\
\hline & & & 1 & $172 \mathrm{~T}_{j \mathrm{v}}=33 \mathrm{~T}_{\mathrm{jm}}$ \\
\hline \multirow[t]{2}{*}{$\mathrm{J}+\mathrm{M}+\mathrm{Ma}$} & 6.75 & 6.76 & 3.2 & $3 \mathrm{~T}_{\text {jma }}=27 \mathrm{~T}_{\mathrm{jm}}$ \\
\hline & 4.79 & 4.8 & & $2 \mathrm{~T}_{j \mathrm{ma}}=20 \mathrm{~T}_{\mathrm{jm}}$ \\
\hline$J+\mathrm{H}+\mathrm{V}+\mathrm{E}$ & 66.53 & 66.7 & 6.33 & $\begin{aligned} 61 \mathrm{~T}_{j \mathrm{e}} & =103 \mathrm{~T}_{\mathrm{jv}} \\
& =271 \mathrm{~T}_{\mathrm{jm}}\end{aligned}$ \\
\hline
\end{tabular}




\section{ACKNOWLEDGEMENT}

Author wishes to thank Dr. (Mrs.) V.Sinha and Shri S.P. Bhatnagar for interesting discussions and reading the manuscript of the paper.

\section{REFERENCES}

[1] Currie, R.G.(1973), Astrophys. \& Space Sc. 20,509.

[2] Eddy, John, A, Proc. Conf. Ancient Sun (1980),p.113, Jr. Geochem. Soc. \& Met. Society, Suppl.13, Pergamon Press.

[3] O.Brien, K. (1979), Jr.Geophys.Res. 84,423.

[4] Solar - Geophysical Data Prompt Reports of NOAA,Feb. (1985), 468 Part 1 .

[5] Stuiver, M. (1980), Nature, 286,868 .

[6] Stuiver, M. and Grootes, P.M., Proc.Conf.Ancient Sun (1980),p.165, Jr.Geoc hem. Soc. \& Met. Soc iet y, Supp1 .13, Pergamon Press.

[7] Stuiver, M. and Quay, P.D. (1980), Science, 207,11.

[8] Verma, S.D. (1985), Space Dynamics and Celestial Mechanics, 143-154(1986) Fd.K.B. Bhatnagar, Publ i shed by D.Reidel Publishing Company. 